\title{
RENAL VASCULAR PERFUSION INDEX IN A CANINE MODEL
}

\author{
Yio-Wha Shau, ${ }^{\dagger}{ }^{\dagger}$ Sun-Hua Pao, ${ }^{* \dagger}$ NaI-Kuan Chou ${ }^{\ddagger}$ King-Jen Chang, ${ }^{\ddagger}$ and \\ JeOU-Jong SHYU ${ }^{\S}$ \\ *Institute of Applied Mechanics, National Taiwan University, Taipei, Taiwan; ${ }^{\dagger}$ Industrial Technology Research \\ Institute, Hsin-Tsu, Taiwan; ${ }^{\ddagger}$ Department of Surgery, National Taiwan University Hospital, National Taiwan \\ University, Taipei, Taiwan; and ${ }^{\S}$ Department of Veterinary Medicine, National Taiwan University, Taipei, Taiwan
}

(Received 15 November 2007; revised 18 June 2008; in final form 27 June 2008)

\begin{abstract}
Decreased renal perfusion plays an important role in the progression toward renal failure. In this study, a novel measure was proposed to quantify renal perfusion using canine model. Serial renal vascular images at different vascular areas including the whole vascular tree, interlobar, arcuate and interlobular vessels were captured. Image processing software was designed to analyze the changes of power Doppler intensity of colored pixels within regions-of-interest (ROI). For a given ROI, the power Doppler vascular index (PDVI) was found to fluctuate with the cardiac cycle. It was also noted that the power Doppler signals generated by arterial vessels have different fluctuating waveforms and different phase compared with the signal derived from venous vessels. A power Doppler correlationmap was developed to differentiate the arteries and veins in the ROI. Using the serial power Doppler images and the derived flow direction information, the interlobular perfusion can be strongly quantified. The renal vascular perfusion index (RVPI) defined as the ratio of PDVI ${ }_{\text {max }}$ versus PDVI $_{\text {min }}$ was significantly higher in the interlobular vessel areas than three other areas for seven healthy dogs. The RVPI resembles the systolic/diastolic (S/D) ratio that commonly reflects arterial hemodynamics. RVPI and power Doppler correlation-map reveal more "dynamic" sense of vascular perfusion and provide a novel approach for the examination of renal function in clinical practice. (E-mail: jjvetmed@ntu.edu.tw) (C) 2008 World Federation for Ultrasound in Medicine \& Biology.
\end{abstract}

Key Words: Ultrasound, Power Doppler vascular index, Renal vascular perfusion index, Interlobular vessel.

\section{INTRODUCTION}

Early detection of renal problems is beneficial to proper medication. During the last decade, both color Doppler and power Doppler sonography have been widely used to evaluate renal functions (Bude et al. 1994; Chen et al. 1998; Clautice-Engle and Jeffery 1997). Unlike computer tomography (CT) scans and magnetic resonance imaging (MRI), color Doppler images, based on the mean Doppler frequency shift induced by blood flow velocity, easily present the overall renal vascular structure, as well as the flow direction and velocity. However, color Doppler is not sensitive in depicting small vessels or vessels with low velocity. Power Doppler sonography is based on the total integrated energy of Doppler shifted echoes and has higher sensitivity for much slower blood flow but it can not address the flow direction or flow velocity (Bude and Rubin

Address correspondence to: Dr. Jeou-Jong Shyu, Comparative Medicine Lab., Department of Veterinary Medicine, National Taiwan University, No.1, Sect. 4, Roosevelt Road, Taipei, Taiwan. E-mail: jjvetmed@ntu.edu.tw
1996; Martinoli et al. 1998; Murphy and Rubin 1997; Rubin et al. 1994; Turetschek et al. 1999).

Lately, a directional power Doppler imaging mode has been implemented in commercially available duplex ultrasound systems (Toshiba, ATL-Philips, GE) in which the positive and negative flows are separated before the estimation of Doppler strength (Claudon et al. 2002). The flow direction is encoded in the power Doppler image with a two-color scale, which may be helpful in some clinical cases.

Bude et al. (1994) used power Doppler sonography to demonstrate a diffuse blush on the renal cortex (region covered by arcuate and interlobular vessels) with normal renal vasculature. Durick et al. (1995) examined the decrease and increase of power Doppler vascular perfusion on the kidney after administration of epinephrine and papaverine, respectively, in swine. Dacher et al. (1996) and Clautice-Engle and Jeffery (1997) identified the renal hypoperfusion with acute pyelonephritis or renal pathology using power Doppler. Martinoli et al. (1996) used graded interlobular vessel signals to evaluate renal transplantation with chronic rejection and Sidhu et 
al. (1999) used power Doppler to show vascular pruning or renal hypoperfusion in acute renal transplant rejection.

Among these studies, power Doppler was used to only collect "static" information on renal vascular perfusion without details on hemodynamics. In this study, utilizing canine models, a novel renal vascular perfusion index (RVPI) and a power Doppler correlation-map are proposed to study the "dynamic" renal vascular perfusion, which would be useful for routine examination of renal vascular perfusion in human patients. "Dynamic" means that changes of blood flow during the cardiac cycle are considered.

\section{MATERIALS AND METHODS}

\section{Animal preparation and ultrasonography}

All animals involved received humane care in compliance with the "Guiding Principles in the Care and Use of Animals" (American Physiological Society, revised 1980), the "Guide for the Care and Use of Laboratory Animals" (NIH publication No. 85-23, revised 1985), and the guideline of the Institutional Animal Care and Use Committee of the National Taiwan University (NTU). Seven healthy mongrel dogs approximately 15 to $20 \mathrm{kgs}$ were used in this study. After sedation with $25 \mathrm{mg}$ of chlorpromazine hydrochloride (Ying-Yuan, Tainan, Taiwan) for $30 \mathrm{~min}$, the dog was anesthetized intravenously with $0.2 \mathrm{~g}$ of thiamylal $(0.5$ $\mathrm{g}$ in $20 \mathrm{ml}$ distilled water) (Citosol; Kyolin, Tokyo, Japan) and maintained at $2.5 \%$ to $4.0 \%$ of 2-bromo-2-chloro-1, 1, 1-trifluoroethane (Fluothane-Zeneca, Macclesfield, Cheshire, UK) administered via an endotracheal tube. The respiration was sustained with a microprocessor Ventilator $7200(\mathrm{Pu}-$ ritan-Bennett, Carlsbad, CA, USA). To monitor the electrocardiogram (ECG), three electrodes were connected on one foreleg and both hind legs. The blood pressure was monitored by an OscilloMate 9300 (Cas Medical System, Branford, CT, USA). During image examination, the respiration rate was adjusted to about 12 cycles per min. For image data collection, the respiration rate was reduced to 6 cycles per min, so that continuous images for more than two to three cardiac cycles could be collected without respiratory interference.

Clinical ultrasound (ATL HDI 5000-Sono CT; Philips, Bothell, WA, USA) with a 5 to $12 \mathrm{MHz}$ linear array transducer (L12-5 38mm) was used in this study. Using power Doppler mode, the ultrasound system was set at a scanning depth of $4.8 \mathrm{~cm}$, with Color Power Angio (CPA) of about $80 \%$ and medium wall filter; the pulsed repetition frequency (PRF) given was about $1000 \mathrm{~Hz}$. With the kidney located at about $2 \mathrm{~cm}$ in depth below the scanner head and with a scanning window of about $2 \times 3$ $\mathrm{cm}^{2}$, the frame rate was about $13 \sim 15$ frames per $\mathrm{s}$.

\section{Data collection and analysis}

Initially a B-mode scan was performed to examine the whole kidney, followed by the pyramid, inter-pyramid areas and cortex scans. Both color Doppler and power Doppler sonograms were used to examine the blood flow in those selected areas and only consecutive pictures without severe movement artifacts were recorded. All the captured power Doppler images were analyzed off-line in DICOM (digital image for communication in medicine) format that contained contiguous 45 images (24-bits color, 640 x 476 pixels), covering about $3 \mathrm{~s}$. In some cases, the interlobar pulsed Doppler images were also collected for comparisons.

The vascular power Doppler signal was analyzed using laboratory-designed (Angiogenesis Research Center, NTU) Renal Vascularity Index Quantification software written in Borland $\mathrm{C}++$ Builder (Borland Software Corporation, Scotts Valley, CA, USA). To correct for breathing and body movements, the viewing window in sequential frames were carefully realigned using twodimensional (2D) image auto-correlation of selected grey-scale land-marks in the sonogram.

Power Doppler vascularity index (PDVI) has been commonly defined as the percentage of color pixels within a region-of-interest (ROI) (Wu et al. 1998; Cheng et al. 1999; Chen et al. 2004):

$$
P D V I^{k}=\frac{1}{A_{R O I}} \cdot \sum_{i=1}^{n x} \sum_{j=1}^{n y} I_{i, j}^{k}(k=1, N)
$$

where superscript $\mathrm{k}$ is the frame index of consecutive images, $\mathrm{N}$ is the total number of frames $(\mathrm{N}=45)$, and $\mathrm{nx}$ and ny are the width and height of the ROI for the i-th row and $\mathrm{j}$-th column, respectively. $A_{R O I}$ is the total number of pixels in the ROI and $\mathrm{I}_{\mathrm{i}, \mathrm{j}}$ is the binary-coded matrix from the digitalized image; where $\mathrm{I}_{\mathrm{i}, \mathrm{j}}=1$ for colored pixels otherwise $\mathrm{I}_{\mathrm{i}, \mathrm{j}}=0$. Figure 1 shows the typical power Doppler sequential images of the renal vascular tree (ROI: $1.00 \mathrm{~cm}$ $x 1.83 \mathrm{~cm}$; nx: 230; ny: 126), which include maximum and minimum PDVI conditions. With a power Doppler imaging frame rate of $13 \mathrm{~Hz}$, the percentage of colored pixels in the ROI (i.e., PDVI value) was found to change with the cardiac cycle (Fig. 1).

Figure 2a shows four typical renal vessel selections for PDVI analysis: (A) the whole vascular tree, (B) interlobar, (C) arcuate and (D) interlobular vessels. The size of ROI should be large enough to enclose the specific vessels of interested and small enough to give an acceptable image frame rate (i.e., $\mathrm{N}>10$ ) for waveform analysis. It should be noted that with the target vessels enclosed an increasing ROI by adding more nonvascular area results in a smaller PDVI value. For organs with uniformly distributed vascular area, PDVI is less affected 


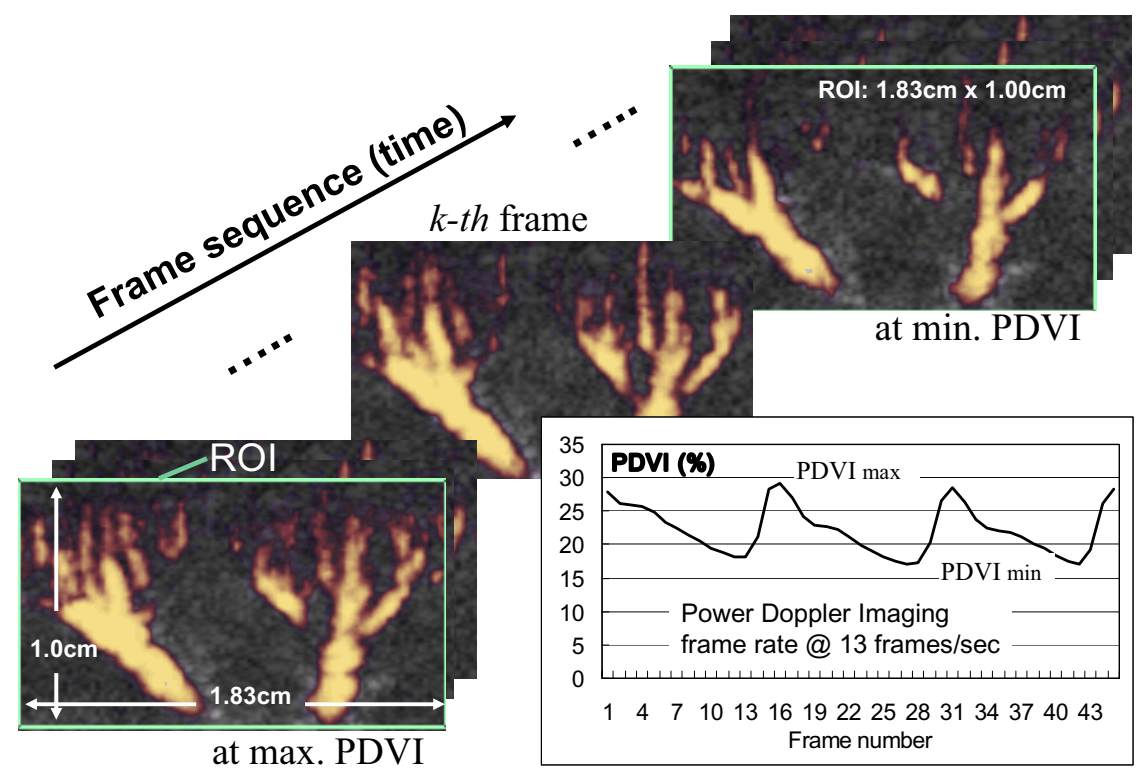

Fig.1. Typical power Doppler vascularity index (PDVI) images of the renal vascular tree, images corresponding to $\mathrm{PDVI}_{\text {max }}$ (bottom-left) and PDVI ${ }_{\text {min }}$ (upper-right). The PDVI waveform analyzed from 45 consecutive power Doppler images is shown in the bottom-right. Region-of-interest (ROI) image size: $1.83 \mathrm{~cm} \times 1.00 \mathrm{~cm}$.

by the size of ROI. It is of interest to know how the size of ROI affects the PDVI values at systole and diastole.

To examine the effect of the ROI size on the renal vascular perfusion level, the ROI were selected and intentionally adjusted not only to enclose the specific vessels of interest but also were standardized to give relatively the same peak PDVI levels (i.e., 20\%, 30\% and $40 \%$, respectively). Thus, focus was on the changes of minimum PDVI values for various settings. At least four data points were carefully collected in each PDVI image analysis for all cases.

Figure $2 b$ shows the corresponding PDVI waveforms of the four vascular areas, and Fig. 2c shows the PDVI waveform normalized against its minimum value $\left(\mathrm{PDVI}_{\min }\right)$ that occurred during cardiac cycle. The normalized PDVI highlights the characteristics of renal vascular perfusion in the cortex area. We defined a renal vascular perfusion index (RVPI) as the ratio of the maximum value $\left(\mathrm{PDVI}_{\max }\right)$ to the minimum value $\left(\mathrm{PDVI}_{\min }\right)$ that resembles the systolic/diastolic (S/D) ratio that is commonly used to quantify arterial resistance or vascular perfusion.

From the hemodynamic point of view, the power Doppler scattering waveforms of arterial vessels differ from that of venous vessels in pulsatility and in phase relationship. The arterial inflow and venous outflow show considerable phase difference that attribute to intravascular compliance properties ( $\mathrm{Li}$ 2004). The vessels can be identified by evaluating a normalized power Doppler strength over time. For that purpose, colorweighted power Doppler vascular index (WPDVI) was used in this analysis by weighting the color matrix, $I_{i, j}$, with its power scatter strength.

$$
W P D V I^{k}=\frac{1}{A_{R O I}} \cdot \sum_{i=1}^{n x} \sum_{j=1}^{n y} C_{i, j}^{k}(k=1, N)
$$

where $\mathrm{C}_{\mathrm{i}, \mathrm{j}}{ }^{\mathrm{k}}$ is the power Doppler scatter strength at the location $(i, j)$ of the image matrix at the $k$-th frame normalized by its maximum power scale (i.e., $\mathrm{C}_{\mathrm{i}, \mathrm{j}}{ }^{\mathrm{k}} \leq 1$ ). For initial reference (i.e., the first iteration $\mathrm{n}=1$ ), the WPDVI waveform $\left(\right.$ WPDVI $^{\mathrm{k}}, \mathrm{k}=1, \mathrm{~N}$ ) of the original ROI was used to calculate the correlation matrix, $R_{i, j}$, with the local waveform of scatter strength $\left(\mathrm{C}_{\mathrm{i}, \mathrm{j}}{ }^{\mathrm{k}}, \mathrm{k} \stackrel{\mathrm{i}}{=}\right.$ $1, \mathrm{~N}$ at pixel $(\mathrm{i}, \mathrm{j}))$.

$R_{i, j}$

$$
=\frac{N \cdot \sum_{k=1}^{N} C_{i, j}^{k} \cdot W P D V I^{k}-\left[\sum_{k=1}^{N} C_{i, j}^{k}\right] \cdot\left[\sum_{k=1}^{N} W P D V I^{k}\right]}{\sqrt{N \cdot \sum_{k=1}^{N}\left(C_{i, j}^{k}\right)^{2}-\left[\sum_{k=1}^{N} C_{i, j}^{k}\right]^{2} \cdot}}
$$

Power Doppler correlation-map $\left(\mathrm{A}_{\mathrm{n}}\right.$ : image for the $\mathrm{n}$-th iteration) was constructed using a neutral green background, the pixels with positive correlation $\left(\mathrm{R}_{\mathrm{i}, \mathrm{j}}>\right.$ 0 ) were marked red and pixels with negative correlation 
(a)
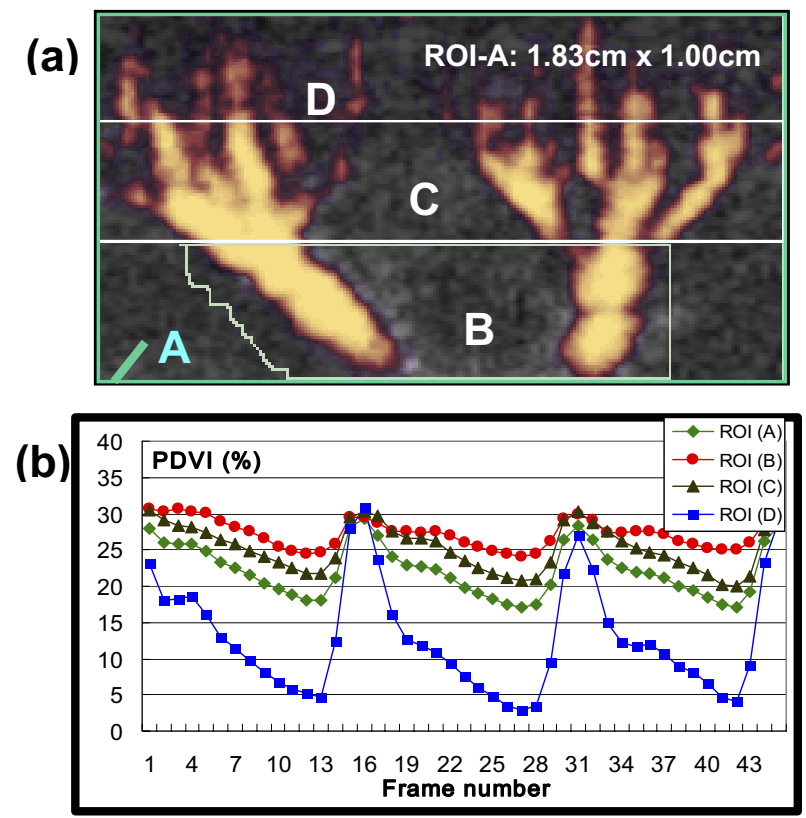

(c)

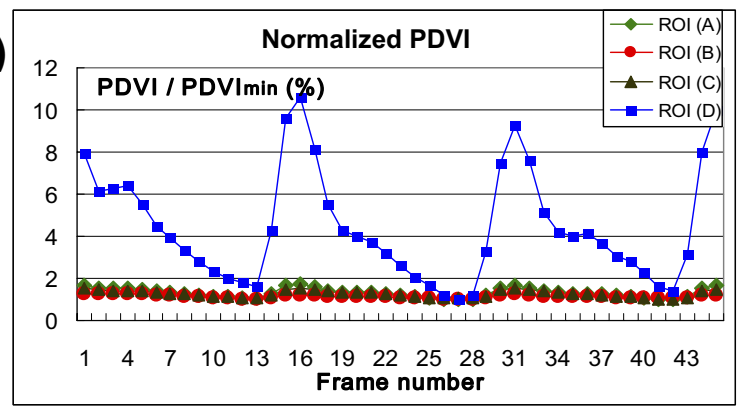

Fig. 2. (a) Typical region-of-interest (ROI) selection for renal power Doppler image; (A) the whole vascular tree, (B) the interlobar vessels, (C) the arcuate vessels and (D) the interlobular vessels; (b) Power Doppler vascularity index (PDVI) curves for the four ROI selections above that were analyzed from 45 consecutive power Doppler images; and (c) normalized PDVI by the corresponding PDVI min $_{\text {. The normalized }}$ PDVI waveform for the interlobular vessels, ROI-(D), has much higher fluctuating amplitudes than that of the other three areas. ROI-A image size: $1.83 \mathrm{~cm}$ x $1.00 \mathrm{~cm}$.

$\left(\mathrm{R}_{\mathrm{i}, \mathrm{j}}<0\right)$ were marked blue. The intensity of the red- or blue-color pixel represents the relative magnitude of correlation $\left(\mathrm{R}_{\mathrm{i}, \mathrm{j}}\right)$. For convenience, a maximum red- or blue-color intensity level of 100 was used in the 24-bits color image.

Since the initial power Doppler correlation-map $\left(A_{n} ; n=1\right)$ was drawn based on the WPDVI waveform that took all pixels of either positive or negative correlations $\left(\mathrm{R}_{\mathrm{i}, \mathrm{j}}\right)$ into account, an iterative process was needed to purify the correlation-map $\left(\mathrm{A}_{\mathrm{n}}\right)$ with the reference-WPDVI waveform that associated with pixels of positive correlation $\left(\mathrm{R}_{\mathrm{i}, \mathrm{j}}>0\right)$ only.

Therefore, the reference-WPDVI waveforms (WP-
$\mathrm{DVI}^{\mathrm{k}}, \mathrm{k}=1, \mathrm{~N}$ ) were iteratively updated and the correlation matrix $\left(\mathrm{R}_{\mathrm{i}, \mathrm{j}}\right)$ between the WPDVI waveform and the local power Doppler scatter waveform $\left(\mathrm{C}_{\mathrm{i}, \mathrm{j}}{ }^{\mathrm{k}}, \mathrm{k}=\right.$ $1, N$ ) was recalculated. The iterative procedure was performed until the percentage of pixel-changes between the new power Doppler correlation-map $\left(A_{n}\right)$ and the previous one $\left(A_{n-1}\right)$ was less than a selected threshold (i.e., $1 \%$ ). Figure 3 shows the flow chart of the procedure for power Doppler correlation-map construction. Typically, the analysis of the correlation-map takes less than 10 iterations to converge and no more than $10 \mathrm{~s}$ overall on a Pentium II personal Computer. We then calculate separately the WPDVI waveform for those pixels with positive correlation (red pixels; $R_{i, j}>0$ ) and the WPDVI waveform for those pixels with negative correlation (blue pixels; $\mathrm{R}_{\mathrm{i}, \mathrm{j}}<0$ ). The converged results of the power Doppler correlation-map are shown in Fig. 4a and the corresponding WPDVI waveforms for both positive (i.e., artery-like) and negative correlations (i.e., veinlike) are plotted on the same figure (Fig. 4b) for comparison.

The statistical significance of the data was determined with the Student's $t$ test; $p<0.05$ was considered to be a statistically significant difference. The results were expressed as means $\pm \mathrm{SD}$ with four data points in each analysis. All statistics were computed by using Microsoft Excel 2000.

\section{RESULTS}

Table 1 shows the PDVI ${ }_{\min }$ and RVPI at the four renal vascular areas, namely (A) the whole vascular tree, (B) interlobar vessels, (C) arcuate vessels and (D) interlobular vessels, where the ROI were selected to give three different levels of $\mathrm{PDVI}_{\max }$ (i.e., about 20\%, 30\% and $40 \%$, respectively) for comparisons. The $p$-values were also given by comparing the results of interlobular vessels (ROI-D) with the other three vascular areas using Student's $t$ test. Table 2 shows the RVPI indices at the four renal vascular areas for the seven experimental animals. In all cases, the RVPI values for the interlobular vessels (ROI-D) were significantly higher than those of the other three areas $(p<0.01)$.

Figure 5 shows the comparisons of (a) the power Doppler image at systole, (b) the calculated power Doppler correlation-map and (c) the color Doppler image at systole of a typical renal vasculature. The area of red-pixels (where $R_{i, j}>0$ ) in Fig. $5 b$ is found to correspond to the renal arteries in Fig. $5 c$ and the blue-pixels $\left(\mathrm{R}_{\mathrm{i}, \mathrm{j}}<0\right)$ is found to correlate with the venous vessels. In Fig. 5d, the pulsed Doppler spectrum measured at the circled area of Fig. 5c revealed a biphasic flow spectrum indicating the collateral structure of interlobar artery and vein, respectively. 


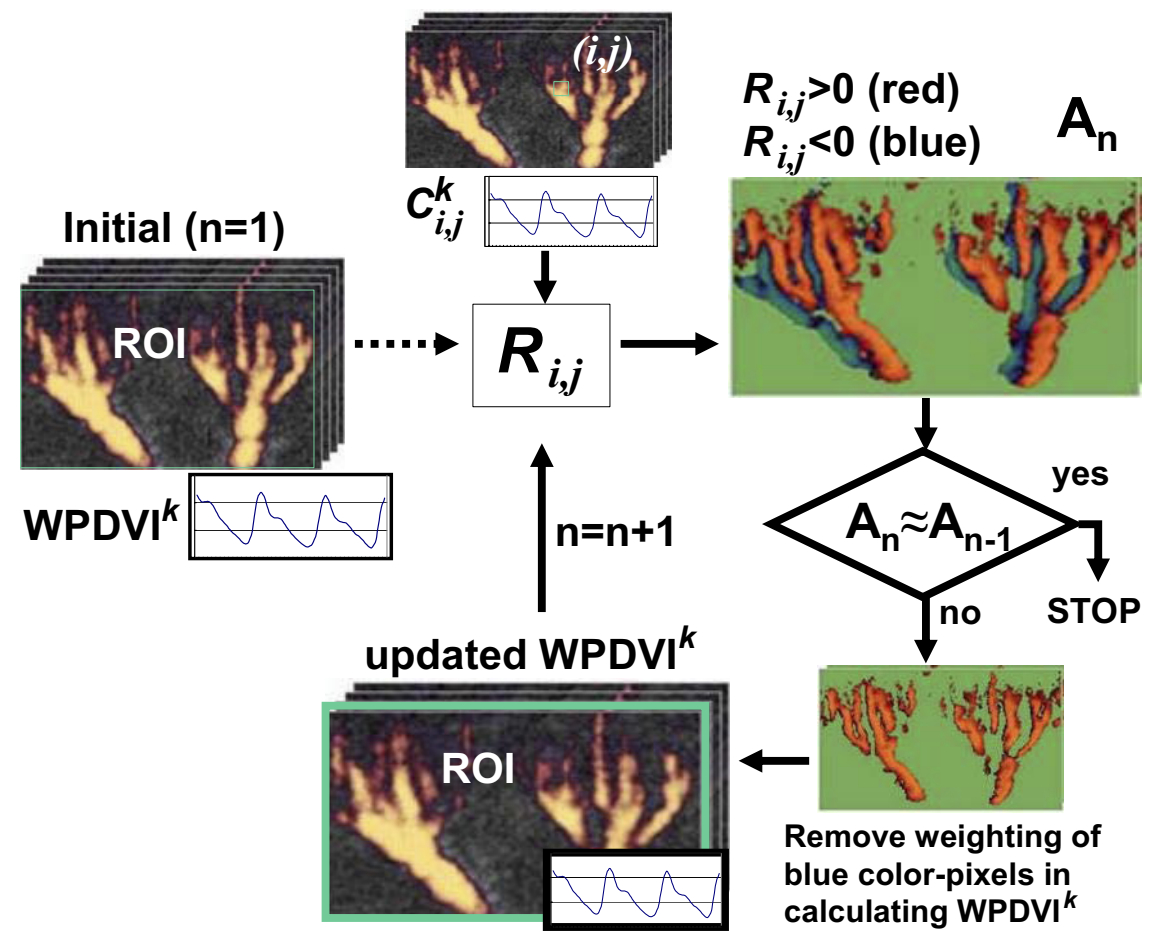

Fig. 3. Schematic flow chart of power Doppler correlation-map analysis. The color-weighted power Doppler vascular index color-weighted (WPDVI) waveform in the region-of-interest (ROI) was calculated for initial reference; the background of the correlation-map was painted green; then the pixels that showed a positive correlation $\left(R_{i, j}>0\right)$ with the initial WPDVI waveform were marked red on the correlation-map $\left(A_{n}\right)$ and the pixels with negative correlations were given a blue color. The reference WPDVI waveform was updated based on the red region only on map $A_{n}$ and then compared with the local $C_{i, j}$ waveforms again. The procedure was repeated iteratively until the percentage of pixel change between correlation-maps $\mathrm{A}_{\mathrm{n}-1}$ and $\mathrm{A}_{\mathrm{n}}$ was less than the required threshold (i.e., $1 \%$ ).

\section{DISCUSSION}

Power Doppler imaging has been widely used to depict the renal cortical perfusion and has been shown to be superior to color Doppler imaging especially in the detection of areas of ischemia or infarction (Chen 1998; Helenon et al. 1998; Kuwa et al. 2004). However, little attention has been paid to the pulsatile characteristics of the power Doppler signal and the link between this and blood vessels hemodynamics (Claudon et al. 2002). Welsh and Fisk (2001) observed the periodic fluctuation of power Doppler signals in fetal renal cortexes and an index of fractional vascular volume was suggested to quantify the renal vascular perfusion. Nagao et al. (2002) also found that the renal power Doppler vascular area pulsated with time and an arterial compliance index was developed to quantify the change in the PDVI waveform caused by hypertension. Using the color Doppler sonographic video, Scholbach et al. (2004) proposed a "dynamic" perfusion intensity index to quantify the renal perfusion in which the mean velocity of color pixels in the ROI were averaged over a cardiac cycle.

Clinical applications of power Doppler imaging are still under investigation, especially for the irregular and tortuous vasculature and technical efforts are needed to separate the arteries from veins on the basis of pulsatility. In the present study, we not only demonstrate the renal PDVI to pulsate over the cardiac cycle but also to correlate the color-weighted WPDVI waveform to the hemodynamics of renal vessels using a power Doppler correlation-map. As seen in Fig. 4, the color-weighted power Doppler flow signals generated by the arterial vessels (which are positively correlated with the overall WPDVI waveform) have higher fluctuating amplitude than those of the venous vessels. The arterial vessels generate a WPDVI waveform, which is nearly opposite in phase with the venous vessels and there appears to be some phase delay in the venous WPDVI waveform (Fig. $4 \mathrm{~b})$. The pulsation of the overall WPDVI or PDVI waveform, which is the sum of arterial vessels and venous vessels, is dominated by arterial signals. Separating the two vascular groups would have a lot of advantages in clinical applications such as tumor hemodynamics.

Consecutively acquired power Doppler images can be used to differentiate the arteries from the veins in the ROI. By using a correlation-map, we can quantify the corresponding vascular perfusion with higher sensitivity and 
(a)

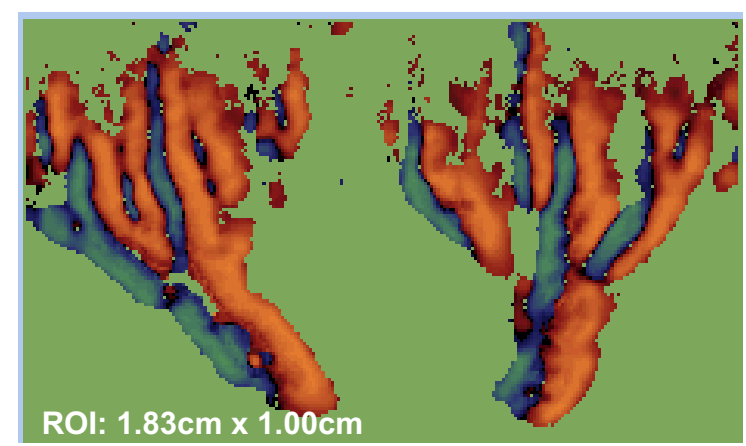

(b)

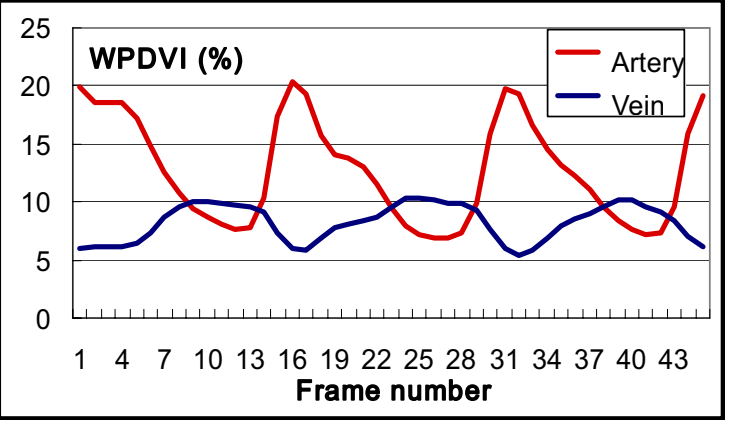

Fig. 4. (a) Converged results of a power Doppler correlationmap. (b) The color-weighted power Doppler vascular index (WPDVI) waveforms for pixels with positive correlation (red: artery-like) and negative correlation (blue: vein-like) for a consecutive 45 frames of the whole renal region-of-interest (ROI) (image size: $1.83 \mathrm{~cm} \times 1.00 \mathrm{~cm}$ ). It appears that the arterial vessel give nearly an opposite waveform than that of the venous vessels.

with flow direction information. The sensitivity of power Doppler imaging in depicting slow flow regions can be further enhanced by using ultrasonic contrast-agents (Wei et al. 2001; Claudon et al. 2002). The directional power Doppler imaging provides only a "freeze" view of the vascular perfusion, whereas the power Doppler correlationmap depicts the pulsating flow patterns and takes the whole cardiac cycle into consideration, which gives more "hemodynamics" aspects.

The physiological relevancy of the correlation-map (Fig. 5b) has been verified via a color Doppler image (Fig. 5c) and strengthened by the Doppler spectrum (Fig. 5d). Comparing Figs. 5a and 5b, it is obvious that the vascular area (colored pixels) in the ROI average over the cardiac cycle is larger than the vascular area observed in systole. There exists several venous flow related power Doppler signals that do not appear in the systolic peak image and, sometimes, the difference in area maybe as high as $20 \%$.

As pointed out earlier, the post-processing time for correlation-map analysis of power Doppler video (with consecutive 45 frames of ROI size $2 \mathrm{~cm} \times 2 \mathrm{~cm}$ ) is relatively fast and typically less than $10 \mathrm{~s}$ including the loading time of images in DICOM format. The present algorithm requires tracing images for at least one heart cycle so the display of a color correlation-map for vessels in moving organs is not truly "real time". However, it is relatively easy to implement in the commercially available ultrasound equipment such as HDI-5000 (ATLPhilips) or LOGIC 700 (GE), which can accommodate advanced image analysis software (i.e., tissue harmonic imaging and three-dimensional imaging).

In Table 1, with various selections of renal vessels, the normalized PDVI waveforms (i.e., $\mathrm{PDVI}^{\mathrm{K}} / \mathrm{PDVI}_{\text {min, }}$, $\mathrm{k}=1, \mathrm{~N})$ in the interlobular area have a higher sensitivity than in the other areas. For various levels of PD$\mathrm{VI}_{\max }$ setting (i.e., $20 \%, 30 \%$ and $40 \%$ ) the $\mathrm{PDVI}_{\min }$ also changed with the PDVI ${ }_{\max }$ in magnitude but the RVPI is less affected. Therefore, the renal vascular perfusion index (RVPI) defined as $\left(\mathrm{PVDI}_{\max } / \mathrm{PVDI}_{\min }\right)$ can emphasize the dynamic vascular perfusion of terminal arteries before entering the glomerular capillaries where blood infiltration takes place. This allows one to detect compromises of terminal vascular perfusion in a variety of disease conditions, such as obstructive lesions occurring in thrombosis, arteriosclerosis or parasite embolism; or compression by adjacent lesions such as granulomas, abscess, tumors, hydronephrosis or renal calculi; or vascular lesions due to renal transplant rejection (Chen et al 1998; Sidhu et al. 1999).

It should be noted that the power Doppler correlation-map analysis requires at least a frame rate of about 10 to extract a good WPDVI waveform for subjects with a heart rate of 72 beats per min. Because power Doppler takes a longer scanning time and is more sensitive to

Table 1. PDVI $_{\max }$, PDVI $_{\min }$ and RVPI indices at different renal vascular areas

\begin{tabular}{lcrcc}
\hline ROI selection* & PDVI $_{\max }$ & \multicolumn{1}{c}{ PDVI $_{\min }$} & RVPI & $p$-value $\dagger$ \\
\hline ROI-A (20\%) & $20.8 \pm 0.2$ & $12.1 \pm 0.1$ & $1.72 \pm 0.00$ & $<0.001$ \\
ROI-B (20\%) & $20.1 \pm 0.2$ & $15.6 \pm 0.2$ & $1.28 \pm 0.01$ & $<0.001$ \\
ROI-C (20\%) & $19.8 \pm 0.5$ & $13.0 \pm 0.3$ & $1.52 \pm 0.01$ & $<0.001$ \\
ROI-D (20\%) & $20.4 \pm 0.5$ & $2.1 \pm 0.2$ & $9.81 \pm 0.73$ & \\
ROI-A (30\%) & $30.1 \pm 0.6$ & $17.6 \pm 0.4$ & $1.71 \pm 0.02$ & $<0.001$ \\
ROI-B (30\%) & $30.7 \pm 0.1$ & $24.1 \pm 0.1$ & $1.28 \pm 0.00$ & $<0.001$ \\
ROI-C (30\%) & $30.6 \pm 0.3$ & $20.1 \pm 0.3$ & $1.52 \pm 0.01$ & $<0.001$ \\
ROI-D (30\%) & $30.4 \pm 1.1$ & $2.9 \pm 0.1$ & $10.7 \pm 0.42$ & \\
ROI-A (40\%) & $40.2 \pm 0.5$ & $23.4 \pm 0.3$ & $1.73 \pm 0.02$ & $<0.001$ \\
ROI-B (40\%) & $40.8 \pm 0.7$ & $31.8 \pm 0.5$ & $1.28 \pm 0.00$ & $<0.001$ \\
ROI-C (40\%) & $40.6 \pm 0.9$ & $26.7 \pm 0.6$ & $1.52 \pm 0.00$ & $<0.001$ \\
ROI-D (40\%) & $40.1 \pm 1.2$ & $3.4 \pm 0.4$ & $11.8 \pm 1.59$ & \\
\hline
\end{tabular}

PDVI = power Doppler vascularity index; RVPI $=$ renal vascular perfusion index; ROI $=$ region-of-interest; $\mathrm{ROI}-\mathrm{A}=$ the whole renal vascular tree; ROI-B = the interlobar vessel area; $\mathrm{ROI}-\mathrm{C}=$ the arcuate vessel area; ROI-D $=$ the interlobular vessel area.

Results were expressed as means \pm SD with four data points collected in each analysis.

* ROI size were intentionally selected to set $\mathrm{PDVI}_{\max }$ at different levels $(20 \%, 30 \%$ and $40 \%)$, respectively, and focus on the changes of $\mathrm{PDVI}_{\min }$. and RVPI.

$\dagger p$-values were given by comparing the results of the three vascular areas with the interlobular vessels (ROI-D) using Student's $t$ test. 
Table 2. RVPI indices at different renal vascular areas collected from seven healthy experimental dogs

\begin{tabular}{lccccccc}
\hline & NO. 1 & NO. 2 & NO. 3 & NO. 4 & NO. 5 & NO. 6 & NO. 7 \\
\hline ROI-A & $1.72 \pm 0.01$ & $1.43 \pm 0.03$ & $1.81 \pm 0.01$ & $1.45 \pm 0.00$ & $1.74 \pm 0.03$ & $1.72 \pm 0.00$ & $1.55 \pm 0.01$ \\
$p$-value* & 0.0023 & 0.01 & $<0.0001$ & 0.00036 & 0.0002 & 0.00027 & 0.0013 \\
ROI-B & $1.34 \pm 0.03$ & $1.06 \pm 0.001$ & $1.11 \pm 0.03$ & $1.06 \pm 0.01$ & $1.28 \pm 0.04$ & $1.14 \pm 0.02$ & $1.18 \pm 0.04$ \\
$p$-value* & 0.0017 & 0.0087 & $<0.0001$ & 0.0003 & 0.00018 & 0.0002 & 0.001 \\
ROI-C & $1.44 \pm 0.01$ & $1.40 \pm 0.04$ & $1.53 \pm 0.06$ & $1.24 \pm 0.02$ & $1.25 \pm 0.05$ & $1.27 \pm 0.08$ & $1.42 \pm 0.11$ \\
$p$-value* & 0.0019 & 0.011 & $<0.0001$ & 0.00031 & 0.00019 & 0.0002 & 0.0015 \\
ROI-D & $6.71 \pm 1.02$ & $7.23 \pm 2.01$ & $5.81 \pm .19$ & $8.40 \pm 0.76$ & $5.51 \pm 0.34$ & $6.20 \pm 0.45$ & $6.57 \pm 0.86$ \\
\hline
\end{tabular}

ROI = region-of-interest; ROI-A = whole renal vascular tree; ROI-B = interlobar vessel area; ROI-C = arcuate vessel area; ROI-D = interlobular vessel area.

Results were expressed as means $\pm \mathrm{SD}$ with four data points collected in each analysis.

* $p$-values were given by comparing the results of the three vascular areas with the interlobular vessels (ROI-D) using Student's $t$ test.

motion artifacts, its clinical application may be difficult in patients who are unable to hold their breath during renal sonography. Because canine kidneys are easier to approach from the body surface, a 5 to $12 \mathrm{MHz}$ linear array transducer with power Doppler mode was sufficient in this study. The human kidneys are anatomically much deeper from the body surface than those in canines so a 3 to $5 \mathrm{MHz}$ convex transducer may be needed for most examinations. In our experience, the resolution of the cortical vasculature was not as good when using a convex transducer.

The RVPI (i.e., $\mathrm{PVDI}_{\max } / \mathrm{PVDI}_{\min }$ ) proposed in this study resembles the ratio of peak systolic flow height to end diastolic flow height (S/D) that is commonly used in reflecting the renal vascular resistance, where the flow velocity quantity is replaced by the PDVI. A lower RVPI in the same renal ROI may reflect a decrease in vascular resistance and an increase in renal perfusion. Similarly, one can also define a new PDVI-based resistive index and pulsatility index by

$$
\begin{aligned}
& \mathrm{RI}^{\prime}=\left(\mathrm{PVDI}_{\max }-\mathrm{PVDI}_{\min }\right) / \mathrm{PVDI}_{\max }, \\
& \mathrm{PI}^{\prime}=\left(\mathrm{PVDI}_{\max }-\mathrm{PVDI}_{\min }\right) / \mathrm{PVDI}_{\mathrm{avg}},
\end{aligned}
$$

The traditional S/D ratio, RI or PI measured using pulse Doppler imaging can only depict the flow at the interlobar vessels and at most down to the size of arcuate vessels. RVPI acquired by power Doppler imaging can
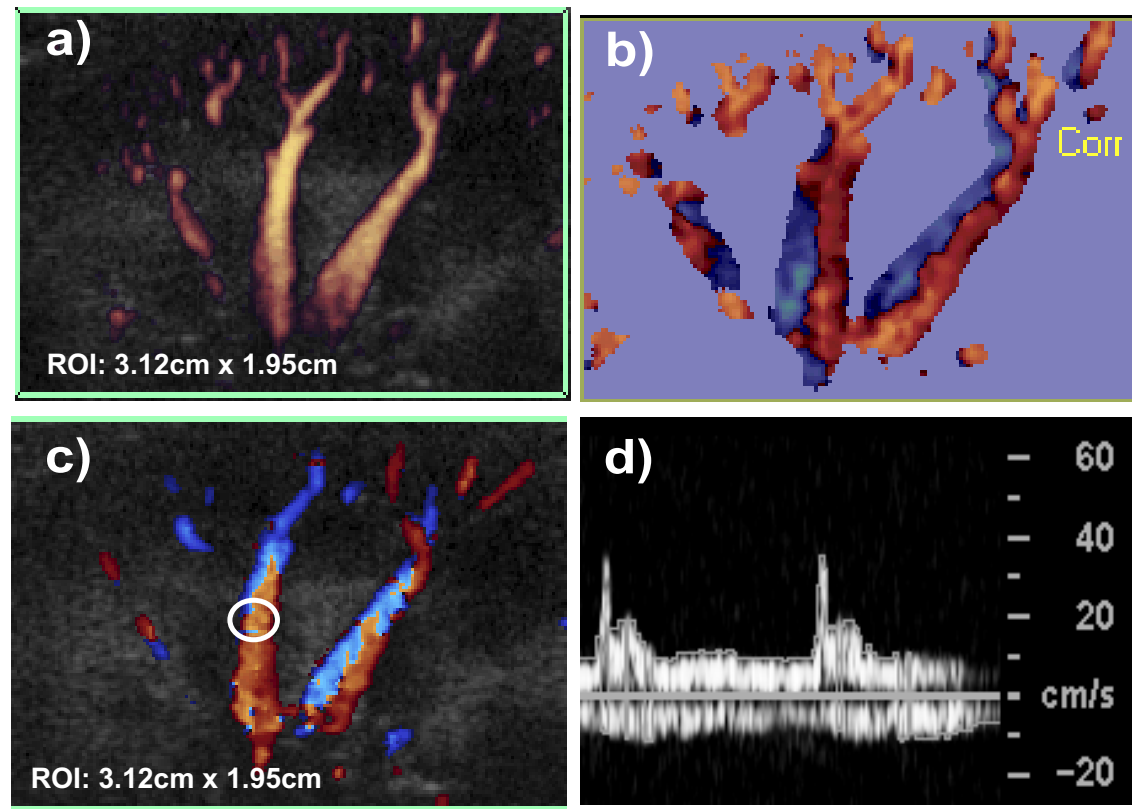

Fig. 5. (a) Another example of a renal power Doppler image; (b) the corresponding correlation-map calculated and (c) a renal color Doppler image taken at the same site. Pulse Doppler was measured at the circled area (interlobar vessels). (d) Pulse Doppler results that revealed a biphasic flow spectra that signified the collateral structure of the interlobar artery and vein respectively. (ROI image size: $3.12 \mathrm{~cm} \mathrm{x} 1.95 \mathrm{~cm}$ ) 
reveal more renal hemodynamics in the cortex area. The dynamic vascular perfusion and vascular resistance information would be useful for monitoring long-term progression of patients with chronic renal diseases.

Recently, we have performed a renal power Doppler study on several healthy volunteers; the PDVI characteristics observed at different renal vascular areas were remarkably similar to those seen in canine models. Also for renal allograft patients, the power Doppler examination with a linear transducer achieves similar results with PDVI analysis. In nephrology clinics, the resistance index (RI) and pulsatility index (PI) are commonly used, but with questionable specificity and sensitivity, to evaluate renal function (Platt 1997; Radermacher et al 2003). This RVPI developed in canine models appears to offer more complete and dynamic information on the renal blood in- and outflow than the traditional methods, thus, demonstrating its potential clinical applications on human patients.

\section{SUMMARY}

Power Doppler imaging has been shown to be more sensitive than color Doppler imaging for vascular perfusion. This study presents a novel power Doppler correlation-map method to differentiate arteries and veins in renal vasculature. We found that the PDVI fluctuates with the cardiac cycle. By normalizing the PDVI value from the sequential power Doppler images with PDVI ${ }_{\text {min }}$ (diastolic) value, we can quantify the vascular perfusion with higher sensitivity and with flow direction information. The renal vascular perfusion index (RVPI) can be used to characterize the dynamic vascular perfusion especially in the interlobular area and is a convenient tool for the examination of renal function in clinics.

Acknowledgments-The authors thank the Minister of Economic Affair (MOEA) for providing the financial support of this project (Contract no. 94-EC-17-A-19-S1-0016) and the Experimental Animal Center of the Department of Veterinary Medicine for assisting animal experiments. The authors also thank for the help for manuscript preparation from Dr. Philip-Leslie Drake and renal ultrasonography from Drs. Shuo-Meng Wang, Tzong-Yann Lee, Chiung-Nien Chen, Meng-Kun Tsai and Shih-Chieh Chueh.

\section{REFERENCES}

Bude RO, Rubin JM, Adler RS. Power versus conventional color Doppler sonography: Comparison in the depiction of normal intrarenal vasculature. Radiology 1994;192:777-780.

Bude RO, Rubin JM. Power Doppler sonography. Radiology 1996;200: $21-23$.

Chen CA, Cheng WF, Lee CN, Su YN, Hsieh CY, Hsieh FJ. Power Doppler vascularity index for predicting the response of neoadjuvant chemotherapy in cervical carcinoma, Acta Obstet Gynecol Scand 2004;83:591-597.
Chen P, Maklad N, Redwine M. Color and power Doppler imaging of the kidneys. World J Uro 1998;16:41-45.

Cheng WF, Lee CN, Chou JS, Chen CA, Chen TM, Shau WY, Hsieh $\mathrm{CY}$, Hsieh FJ. Vascularity index as a novel parameter for the in vivo assessment of angiogenesis in patients with cervical carcinoma. Cancer 1999;85:651-657.

Claudon M, Tranquart F, Evans DH, Lefevre F, Correas JM. Advances in ultrasound. Eur Radiol 2002;12:7-18.

Clautice-Engle T, Jeffery RB Jr. Renal hypoperfusion: Value of power Doppler imaging. AJR 1997;168:1277-1231.

Dacher J-N, Pfister C, Monroc M, Eurin D, Dosseur PL. Power Doppler sonographic pattern of acute pyelonephritis in children: Comparison with CT. AJR 1996;166:1451-1455.

Durick JE, Winter TC, Schmiedl UP, Cyr DR, Starr FL, Mack LA Renal perfusion: Pharmacologic changes depicted with power Doppler US in an animal model. Radiology 1995;197:615-617.

Helenon O, Correas JM, Chabriais J, Boyer JC, Melki P, Moreau JF. Renal vascular Doppler imaing: Clinical benefits of power mode. Radiographics 1998;18:1441-1454.

Kuwa T, Cancio LC, Sondeen JL, Matylevich N, Jordan BS, McManus AT, Goodwin CW. Evaluation of renal cortical perfusion by noninvasive power Doppler ultrasound during vascular occlusion and reperfusion. J Trauma 2004;56:618-624.

Li J K-J. Dynamics of the vascular system. Series on bioengineering and biomedical engineering. Vol. 1. Singapore: World Scientific Publisher Co. Pte. Ltd., 2004.

Martinoli C, Crespi G, Bertolotto M, Rollandi GA, Rosenberg I, Pretolesi F, Derchi LE. Interlobular vasculature in renal transplants: A power Doppler US study with MR correlation. Radiology 1996;200:111-117.

Martinoli C, Derchi LE, Rizzatto G, Solbiati L. Power Doppler sonography: General principles, clinical applications, and future prospects. Eur Radiol 1998;8:1224-1235.

Murphy KJ, Rubin JM. Power Doppler: It's a good thing. Semin Ultrasound CT MRI 1997;18:13-21.

Nagao M, Murase K, Saeki H, Mochizuki T, Sugata S, Ikezoe J. Pulsating renal blood flow distribution measured using power Doppler ultrasound: Correlation with hypertension. Hypertens Res 2002;25:697-702.

Platt JF. Doppler ultrasound of the kidney. Semin Ultrasound CT MRI 1997;18:22-32.

Radermacher J, Mengel M, Ellis S, Stuht S, Hiss M, Schwarz A, Eisenberger U, Burg M, Luft FC, Gwinner W, Haller H. The renal arterial resistance index and renal allograft survival. N Engl J Med 2003;349:115-124.

Rubin JM, Bude RO, Carson PL, Bree RL, Adler RS. Power Doppler US: A potentially useful alternative to mean frequency-based color Doppler US. Radiology 1994;190:853-856.

Scholbach T, Dimos I, Scholbach J. A new method of color Doppler perfusion measurement via dynamic sonographe signal quantification in renal parenchyma. Nephron Physiol 2004;96:99-104.

Sidhu MK, Gambhir S, Jeffery RB Jr, Sommer FG, Li KCP, Krieger NR, Alfreg EJ, Scandling JD. Power Doppler imaging of acute renal transplant rejection. J Clin Ultrasound 1999;27:171-175.

Turetschek K, Kollmann C, Doffner R, Wunderbaldinger P, Mostbeck G. Amplitude-code color Doppler: Clinical applications. Eur Radiol 1999;9:115-121.

Wei K, Le E, Bin JP, Coggins M, Thorpe J, Kaul S. Quantification of renal blood flow with contrast-enhanced ultrasound. JACC 2001; 37:1135-1140.

Welsh AW, Fisk NM. Picture of the month. Assessment of fetal renal perfusion by power Doppler digital analysis. Ultrasound Obstet Gynecol 2001;17:89-91.

Wu CH, Ahang YL, Hsu WC, KO JY, Sheen TS, Hseih FJ. Usefulness of Doppler spectral analysis and power Doppler sonography in the differentiation of cervical lymphadenopathies. Am J Roentgenol 1998;171:503-509 